\title{
Полупроводниковый сенсор термоэлектрического однофотонного детектора для регистрации излучения ближнего ИК диапазона
}

\author{
(C) А.А. Кузанян \\ Институт фризических исследований Национальной академии наук Армении, \\ 0203 Аштарак, Армения \\ E-mail: astghik.kuzanyan@gmail.com
}

Поступила в Редакцию 26 мая 2020 г.

В окончательной редакции 28 ноября 2020 г.

Принята к публикации 28 ноября 2020 г.

\begin{abstract}
Предложена конструкция четырехслойного чувствительного элемента однофотонного термоэлектрического детектора с полупроводниковым $\mathrm{FeSb}_{2}$ сенсором. Методом компьютерного моделирования изучены процессы распространения тепла в чувствительном элементе после поглощения фотона. Расчеты проводились на основе уравнения распространения тепла из ограниченного объема с использованием трехмерного матричного метода для дифференциальных уравнений. Временны́е зависимости амплитуды сигнала детектора рассчитывались для различных толщин слоев чувствительного элемента и определялись следующие параметры: задержка сигнала, временно́й джиттер, максимальное значение сигнала, время достижения максимального сигнала, время затухания и скорость счета. Обосновано, что детектор с таким чувствительным элементом может обеспечить эффективность детектирования $>95 \%$ для фотонов ближней ИК области. Одновременно достигается терагерцовая скорость счета.
\end{abstract}

Ключевые слова: полупроводниковый сенсор, однофотонный детектор, компьютерное моделирование, задержка сигнала, время достижения максимального сигнала.

DOI: $10.21883 /$ FTP.2021.04.50735.9450

\section{1. Введение}

В последние годы наблюдается бурное развитие исследований устройств, способных излучать и регистрировать одиночные фотоны. Однофотонные детекторы (ОД) используются в различных областях науки и техники. Они наиболее востребованы в астрофизике, физике высоких энергий, лазерной спектроскопии, метрологии, медицинском приборостроении, телекоммуникационных системах, устройствах квантовой обработки информации и т.д. [1]. Важнейшими характеристиками ОД являются эффективность детектирования, скорость счета, энергетическое разрешение, скорость темнового счета, спектральный диапазон детектирования и рабочая температура [2,3].

Классические ОД, фотоумножители и полупроводниковые лавинные диоды, имеют ряд недостатков: большие размеры, низкую эффективность детектирования и скорость счета $[4,5]$. Сверхпроводящие ОД имеют более высокие характеристики [6]. На сегодняшний день рекордными характеристиками обладают сверхпроводящие нанопроволочные детекторы [7,8]. Недостатками сверхпроводящих детекторов являются жесткие требования к условиям работы и сложная конструкция сенсора.

Конкуренцию вышеперечисленным ОД может составить термоэлектрический однофотонный детектор (ТОД), физическая концепция которого была предложена в 2000 году группой ученых из США и Армении [9]. Главными преимуществами ТОД являются отсутствие строгих требований к условиям работы и простая конструкция чувствительного элемента [10]. Теоретические оценки показали, что термоэлектрический детектор может иметь более высокие характеристики, чем некоторые сверхпроводящие [11]. Характеристики однослойной и трехслойной конструкции чувствительного элемента ТОД были подробно рассмотрены методом компьютерного моделирования [12-19]. Исследования показали, что использование в качестве термоэлектрического сенсора гексаборидов церия $\mathrm{CeB}_{6}[12,13]$ и лантана церия $(\mathrm{La}, \mathrm{Ce}) \mathrm{B}_{6}[14,15]$ может обеспечить высокую скорость счета и энергетическое разрешение ТОД при 9 и $0.5 \mathrm{~K}$ соответственно. Перспективным материалом сенсора ТОД является также обладающий при низких температурах гигантским коэффициентом Зеебека сильно коррелированный полупроводник $\mathrm{FeSb}_{2}[16,17]$. Для повышения эффективности детектирования была предложена четырехслойная конструкция чувствительного элемента ТОД с антиотражающим слоем $[18,19]$.

Цель данной работы - определение методом компьютерного моделирования оптимальной конструкции четырехслойного чувствительного элемента ТОД с $\mathrm{FeSb}_{2}$ сенсором для регистрации излучения в ближней ИК области. Рассмотрены особенности распространения тепла в чувствительном элементе ТОД после поглощения одиночных фотонов с длиной волны 1550 нм (0.8 эВ) и 1310 нм (0.95 эВ). Такое излучение используется в телекоммуникационных системах и устройствах квантовой обработки информации. 
Таблица 1. Параметры используемых материалов при $9 \mathrm{~K}$

\begin{tabular}{|c|c|c|c|c|}
\hline \multirow{2}{*}{ Параметр } & \multicolumn{4}{|c|}{ Материал } \\
\hline & $\mathrm{SiO}_{2}$ & $\mathrm{FeSb}_{2}$ & $\mathrm{~W}$ & $\mathrm{Al}_{2} \mathrm{O}_{3}$ \\
\hline 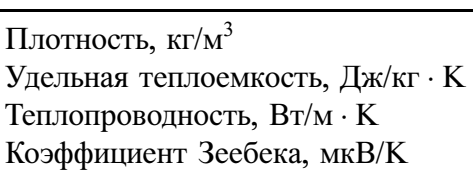 & $\begin{array}{c}2650 \\
1.5[21] \\
0.1[22]]\end{array}$ & $\begin{array}{c}8170 \\
0.0133[23] \\
500[23] \\
-4200[24]\end{array}$ & $\begin{array}{c}19250 \\
0.187[25] \\
9680[26]\end{array}$ & $\begin{array}{c}4000 \\
0.0588[27] \\
300[28]\end{array}$ \\
\hline
\end{tabular}

\section{2. Методика компьютерного моделирования}

В данном параграфе рассмотрены особенности компьютерного моделирования процессов распространения тепла в чувствительном элементе ТОД после поглощения фотона. Подробности использованных подходов и приближений приведены в [20]. Уравнение распространения тепла из ограниченного объема можно представить в виде

$$
\rho c \partial T / \partial t+\partial q_{x} / \partial x+\partial q_{y} / \partial y+\partial q_{z} / \partial z=0,
$$

где $\rho$ - плотность теплопроводящего материала, $c$ удельная теплоемкость, $q_{x}, q_{y}$ и $q_{z}$ - проекции вектора плотности распространения тепла на соответствующие оси, задаваемые уравнениями

$$
q_{x}=-\lambda \partial T / \partial x, q_{y}=-\lambda \partial T / \partial y, q_{z}=-\lambda \partial T / \partial z .
$$

В этих уравнениях $\lambda-$ коэффициент теплопроводности. Мы не рассматриваем процесс конверсии энергии фотона в тепло. Рассматриваются процессы в чувствительном элементе ТОД после того, как фотон термализовался и выделенное им тепло заняло определенный объем в поглотителе. Этот момент является началом рассматриваемых процессов. В расчетах используется трехмерный матричный метод для дифференциальных уравнений в частных производных.

Моделирование процессов распространения тепла после поглощения фотона осуществлялось следующим образом. Весь объем чувствительного элемента делился на ячейки с размерами $\Delta x, \Delta y$ и $\Delta z$. Чем меньше объем ячейки, чем больше их число, тем точнее, но продолжительнее расчеты. Начальная температура всех ячеек чувствительного элемента ТОД принималась равной рабочей температуре $(T)$. В поглотителе выбиралась ячейка, в которой поглощается фотон. Температура данной ячейки задается уравнением $T_{0}=T+\Delta T$, где $\Delta T=E / V \rho c, E-$ энергия фотона, $V$ - объем ячейки, $\rho$ и $c$ - плотность и удельная теплоемкость материала поглотителя. В результате расчетов определяется временна́я зависимость изменения температуры в различных участках чувствительного элемента и рассчитываются основные характеристики детектирования фотона с заданной энергией: максимальная разница температур на границах термоэлектрического сенсора $\left(\Delta T_{m}\right)$, время достижения максимума $\left(t_{m}\right)$, возникающее на сенсоре максимальное электрическое напряжение $\left(V_{m}=S \Delta T_{m}\right.$, где $S$ - коэффициент Зеебека сенсора), время спада температуры до фонового значения $\left(t_{b}=0.1 \mathrm{MK}\right)$ и скорость счета $\left(R=1 / t_{b}\right)$. В эксперименте факт поглощения фотона должен подтверждаться появлением на сенсоре электрического напряжения. Регистрация максимального значения и длительности этого сигнала позволит определить энергию поглощенного фотона и какую скорость счета может обеспечить детектор. Поскольку генерируемое на термоэлектрическом сенсоре напряжение находится в прямой зависимости от коэффициента Зеебека, а для достижения большого значения соотношения сигнала к шуму необходимо спуститься в область низких температур, то в конструкции чувствительного элемента ТОД необходимо использовать материалы с высоким коэффициентом Зеебека при низких температурах. Исходя из этих соображений мы выбрали в качестве материала сенсора чувствительного элемента ТОД коррелированный полупроводник $\mathrm{FeSb}_{2}$.

В настоящей работе рассматривается четырехслойный чувствительный элемент ТОД следующей конструкции. На подложке из сапфира $\left(\mathrm{Al}_{2} \mathrm{O}_{3}\right)$ располагаются последовательно теплоотвод $(\mathrm{W})$, сенсор $\left(\mathrm{FeSb}_{2}\right)$, поглотитель $(\mathrm{W})$ и антиотражающий слой $\left(\mathrm{SiO}_{2}\right)$. Коэффициент Зеебека соединения $\mathrm{FeSb}_{2}$ достигает максимального значения при $9 \mathrm{~K}$. В наших исследованиях именно эта температура выбрана в качестве рабочей температуры детектора. Значения использованных в расчетах физических величин при данной температуре приведены в табл. 1. В расчетах используются также полученные экспериментально температурные зависимости теплоемкости и теплопроводности.

Чтобы добиться высокой эффективности детектирования необходимо обеспечить высокую оптическую связь чувствительного элемента ТОД с регистрируемым излучением, высокую вероятность поглощения попавшего в чувствительный элемент фотона и высокую вероятность регистрации уже поглощенного фотона. Антиотражающий слой $\mathrm{SiO}_{2}$ в конструкции чувствительного элемента ТОД, как и в сверхпроводящих детекторах [29], должен обеспечить высокую оптическую связь излучения ближнего ИК диапазона с чувствительным элементом ТОД. Добиться высокой вероятности поглощения фотонов в поглотителе чувствительного элемента ТОД можно выбором материала поглотителя и его опти- 
мальной толщины. Коэффициент поглощения излучения с длиной волны 1550 и 1305 нм в вольфраме равен $1.1667 \cdot 10^{6}$ и $1.2908 \cdot 10^{6} \mathrm{~cm}^{-1}$ [30]. Используя закон Бугера-Ламберта нетрудно посчитать, что фотоны с энергией 0.8 и 0.95 эВ поглотятся в вольфраме толщиной 0.05 мкм с вероятностью 99.7 и 99.8\%. Поэтому в наших расчетах мы будем рассматривать чувствительные элементы ТОД, толщина поглотителя которых $\geq 0.05$ мкм. Высокая вероятность регистрации уже поглощенного фотона обеспечивается выбором материала сенсора и оптимальной конструкции чувствительного элемента. Эти факторы определяют величину получаемого сигнала и его отношение к уровню шума. Сигнал детектора на уровне микровольтов и десятикратное отношение сигнала к шуму могут гарантировать высокую эффективность регистрации поглощенного фотона.

\section{3. Результаты и их обсуждения}

Нами рассмотрены особенности распространения тепла после поглощения фотонов с энергией 0.8 и 0.95 эВ в центре поверхности четырехслойного чувствительного элемента $\mathrm{SiO}_{2} / \mathrm{W} / \mathrm{FeSb}_{2} / \mathrm{W} / \mathrm{Al}_{2} \mathrm{O}_{3}$ с площадью $10 \times 10$ мкм. Толщина антиотражающего слоя и теплоотвода во всех расчетах составляла 0.1 мкм. Толщина поглотителя и сенсора варьировалась. Рассматривалось поглощение фотонов только в центре поверхности чувствительного элемента, так как ранее нами было показано, что такие характеристики чувствительного элемента, как максимальная разность температур на сенсоре и время спада сигнала до фонового значения, не зависят от области термализации фотона на поверхности поглотителя трехслойного и четырехслойного чувствительного элемента ТОД [19].

\section{а. Поглощение фотонов с энергией 0.8 эВ}

Использованная методика расчета распространения тепла в чувствительном элементе ТОД позволяет определять температуру в любой области чувствительного элемента через любой промежуток времени начиная с момента поглощения фотона. Наше внимание было сосредоточено на регистрации изменения температуры на границах поглотитель-сенсор (A), сенсор-теплоотвод $(B)$ и теплоотвод-подложка $(C)$, данным с которых присвоены индексы $a, b$ и $c$ соответственно. Второй индекс параметра показывает, на каком расстоянии от центра данной границы он регистрируется. Данным с центральных областей слоев чувствительного элемента соответствует индекс 0. Для выявления характеристик детектора нами рассматривались временны́ зависимости температуры в зоне термализации фотона $\left(T_{0}\right)$, непосредственно под зоной термализации фотона на границе $A\left(T_{a 0}\right)$, на границе $B\left(T_{b 0}\right)$, на границе $C\left(T_{c 0}\right)$, а также на границе $A$ на отрезке 1 мкм

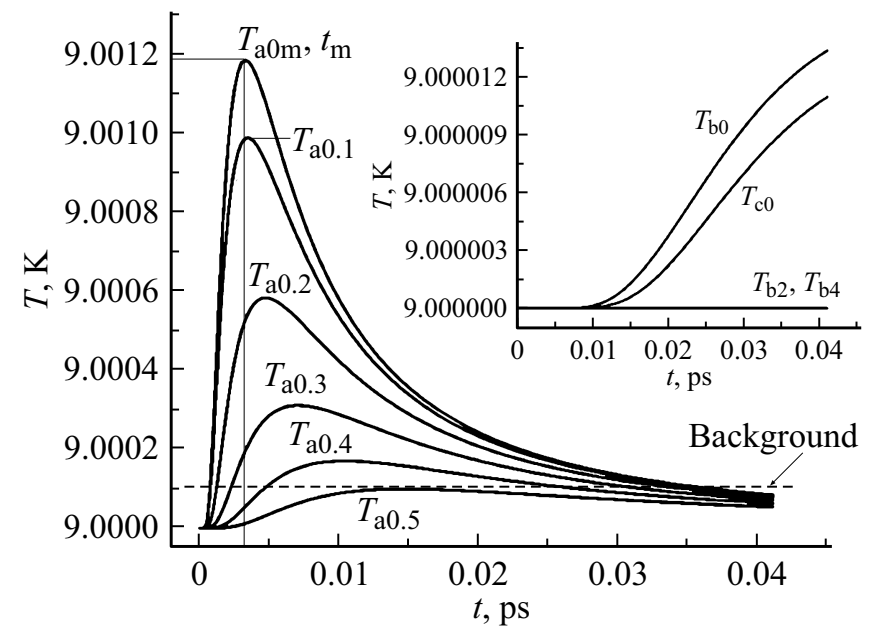

Рис. 1. Временна́я зависимость изменения температуры на различных участках границы поглотитель-сенсор после поглощения фотона с энергией 0.8 эВ в чувствительном элементе с толщинами поглотителя 0.2 мкм и сенсора 1 мкм. На вставке представлены графики временно́й зависимости температуры на границе сенсор-теплоотвод и теплоотвод-подложка.

от центра с шагом 0.1 мкм $\left(T_{a 0.1}, T_{a 0.2}, \ldots, T_{a 1}\right.$ - это температуры на границе поглотитель-сенсор на расстоянии от центра $0.1,0.2, \ldots, 1$ мкм соответственно) и на границе $B$ на расстоянии от центра 2 мкм $\left(T_{b 2}\right)$ и 4 мкм $\left(T_{b 4}\right)$.

Как изменяется температура на границе $A$ после поглощения фотона в чувствительном элементе ТОД с толщиной поглотителя и сенсора 0.2 и 1 мкм показано на рис. 1. Можно видеть, что максимальная температура превосходит значение фона более чем в 10 раз и достигается за время $t_{m}$ непосредственно под зоной термализации фотона $\left(T_{a 0 m}\right)$. Чем дальше от центра находится рассматриваемая область, тем ниже максимум временно́й зависимости температуры и тем позже он достигается. Температура спадает до фонового значения позже всего также в центре границы $A$ и, как мы увидим далее, именно это время определяет параметры $t_{b}$ и $R$.

На вставке к рис. 1 видно, что за время спада температуры на границе $A$ до значения фона на границе $B$ наблюдается подъем температуры $T_{b 0}$. Также растет температура $T_{c 0}$. Однако их значения намного ниже фона, а роста температур $T_{b 2}$ и $T_{b 4}$ вообще не происходит. Сигнал детектора пропорционален максимальной разнице температур на границах $A$ и $B$. В рассматриваемом случае, когда минимальная температура на границе $B$ равна $9 \mathrm{~K}$, возникающий на сенсоре сигнал будет определяться величиной $T_{a 0}$ и задаваться формулой $V=S\left(T_{a 0}-9 \mathrm{~K}\right)$. Параметры $T_{a 0 m}, t_{m}, V_{m}, t_{b}$ и $R$ также будут определяться зависимостью $T_{a 0}(t)$.

Теперь рассмотрим, какими характеристиками будут обладать ТОД при различных значениях толщин поглотителя и сенсора. Переменные параметры чувствитель- 
Таблица 2. Характеристики чувствительного элемента $\mathrm{SiO}_{2} / \mathrm{W} / \mathrm{FeSb}_{2} / \mathrm{W} / \mathrm{Al}_{2} \mathrm{O}_{3}$ при поглощении фотонов с энергией 0.8 эВ

\begin{tabular}{c|c|c|c|c|c|c|c|c}
\hline № & $Z_{2}$, мкм & $Z_{3}$, мкм & $T_{a 0 m}, \mathrm{~K}$ & $T_{b 0 m}, \mathrm{~K}$ & $T_{b 2 m}, \mathrm{~K}$ & $T_{c 0 m}, \mathrm{~K}$ & $t_{m}$, пс & $t_{b}$, пс \\
\hline 1 & 0.2 & 1 & 9.00118 & 9.00001 & 9.00000 & 9.00001 & 0.00330 & 0.03684 \\
2 & 0.2 & 0.5 & 9.00118 & 9.00007 & 9.00000 & 9.00006 & 0.00330 & 0.03768 \\
3 & 0.2 & 0.1 & 9.00120 & 9.00061 & 9.00000 & 9.00042 & 0.00339 & 0.05472 \\
4 & 0.2 & 0.05 & 9.00127 & 9.00092 & 9.00000 & 9.00061 & 0.00357 & 0.05952 \\
5 & 0.1 & 1 & 9.00421 & 9.00002 & 9.00000 & 9.00002 & 0.00126 & 0.03456 \\
6 & 0.1 & 0.5 & 9.00421 & 9.00011 & 9.00000 & 9.00009 & 0.00126 & 0.03642 \\
7 & 0.1 & 0.1 & 9.00422 & 9.00166 & 9.00000 & 9.00096 & 0.00129 & 0.05778 \\
8 & 0.1 & 0.05 & 9.00436 & 9.00281 & 9.00000 & 9.00148 & 0.00135 & 0.06288 \\
9 & 0.05 & 1 & 9.00974 & 9.00002 & 9.00000 & 9.00002 & 0.00042 & 0.03282 \\
10 & 0.05 & 0.5 & 9.00974 & 9.00014 & 9.00000 & 9.00011 & 0.00042 & 0.03498 \\
11 & 0.05 & 0.1 & 9.00974 & 9.00305 & 9.00000 & 9.00152 & 0.00042 & 0.05844 \\
12 & 0.05 & 0.05 & 9.00985 & 9.00559 & 9.00000 & 9.00248 & 0.00045 & 0.06294
\end{tabular}

ного элемента $\mathrm{SiO}_{2} / \mathrm{W} / \mathrm{FeSb}_{2} / \mathrm{W} / \mathrm{Al}_{2} \mathrm{O}_{3}$ и результаты компьютерного моделирования процессов распространения тепла после поглощения фотона с энергией 0.8 эВ приведены в табл. 2. В таблице приведены номер расчета (№), толщина поглотителя $\left(Z_{2}\right)$, толщина сенсора $\left(Z_{3}\right)$ и данные компьютерного моделирования: $T_{a 0 m}, T_{b 0 m}, T_{b 2 m}$, $T_{c 0 m}, t_{m}$ и $t_{b}$. Расчеты проводились при значениях толщины поглотителя $0.2,0.1,0.05$ мкм и толщины сенсора 1 , $0.5,0.1,0.05$ мкм. Температура $T_{b 2 m}$ во всех расчетах не превышает начальную температуру $9 \mathrm{~K}$, следовательно, для всех рассмотренных конфигураций чувствительного элемента характеристики ТОД будет определять зависимость $T_{a 0}(t)$.

В первых 4 строках таблицы приведены результаты расчетов для чувствительных элементов с толщиной $Z_{2}=0.2$ мкм и толщиной $Z_{3}$, уменьшающейся от 1 до 0.05 мкм. Данные расчета № 1 соответствуют представленным на рис. 1 зависимостям $T(t)$. Можно видеть, что на границе $A$ достигается максимальная температура $9.00118 \mathrm{~K}$ за время 0.0033 пс, а время спада сигнала до фонового значения составляет 0.03684 пс. Значения параметров $T_{b 0 m}$ и $T_{c 0 m}$ на порядок ниже фонового значения. С уменьшением толщины сенсора значения $T_{a 0 m}$ несколько увеличиваются, тогда как параметры $T_{b 0 m}$ и $T_{c 0 m}$ увеличиваются значительно. Последнее понятно, так как с уменьшением толщины сенсора до теплоотвода и подложки непосредственно под зоной термализации фотона, не успевая рассеиваться в стороны, доходит большее количество тепла. Временны́е параметры $t_{m}$ и $t_{b}$ с уменьшением $Z_{3}$ увеличиваются, причем $t_{b}$ увеличивается значительно.

Следующие 4 расчета с номерами 5-8 соответствуют чувствительному элементу ТОД с толщиной поглотителя 0.1 мкм. Из представленных в таблице данных сразу же привлекают внимание значительно более высокие (по сравнению с первой четверкой) значения параметров $T_{a 0 m}, T_{b 0 m}$ и $T_{c 0 m}$. Параметр $t_{m}$ с уменьшением $Z_{3}$ несколько увеличивается, но значительно меньше, чем для $Z_{2}=0.2$ мкм. Параметр $t_{b}$ с уменьшением $Z_{3}$ увеличивается, однако, если для $Z_{3}=1$ мкм он несколько

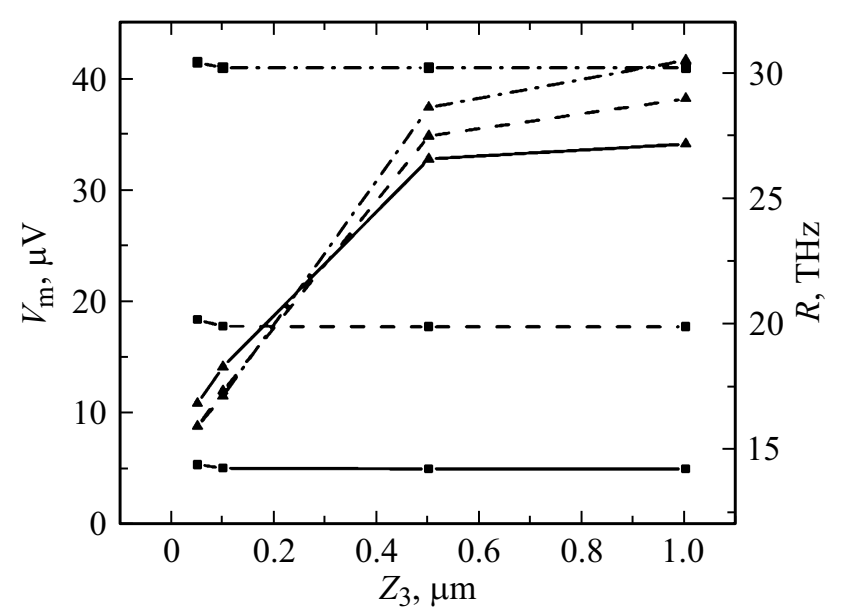

Рис. 2. Зависимость максимального напряжения на сенсоре (квадраты) и скорости счета (треугольники) от толщины сенсора $\left(Z_{3}\right)$ для толщин поглотителя 0.2 мкм (сплошная линия), 0.1 мкм (пунктирная линия) и 0.05 мкм (штрихпунктирная линия).

меньше, то для $Z_{3}=0.05$ мкм он больше, чем в предыдущей четверке расчетов. Основным результатом данной серии расчетов надо считать значительное увеличение температуры $T_{a 0 m}$.

Еще более высокий сигнал можно ожидать от чувствительных элементов с толщиной поглотителя 0.05 мкм (расчеты 9-12). Из представленных в последних 4 строках таблицы данных следует, что параметры $T_{a 0 m}, T_{b 0 m}$ и $T_{c 0 m}$ выше, чем в предыдущих расчетах. Параметр $t_{m}$ принимает значения меньше фемтосекунды. Параметр $t_{b}$ существенных изменений не претерпевает.

Основываясь на данных табл. 2, рассчитаны значения возникающего на чувствительном элементе максимального напряжения $V_{m}$ и скорости счета $R$. Зависимость этих параметров от толщины термоэлектрического сенсора для различных толщин поглотителя приведена на рис. 2. Можно видеть, что регистрируемый в эксперименте сигнал детектора мало изменяется с изменением 
Таблица 3. Характеристики чувствительного элемента $\mathrm{SiO}_{2} / \mathrm{W} / \mathrm{FeSb}_{2} / \mathrm{W} / \mathrm{Al}_{2} \mathrm{O}_{3}$ при поглощении фотонов с энергией 0.95 эВ

\begin{tabular}{c|c|l|l|l|l|l|l|c}
\hline № & $Z_{2}$, мкм & $Z_{3}$, мкм & $h$, мкм & $T_{a 0 m}, \mathrm{~K}$ & $T_{b 0 m}, \mathrm{~K}$ & $T_{c 0 m}, \mathrm{~K}$ & $t_{m}$, пс & $t_{b}$, пс \\
\hline 13 & 0.2 & 1 & 0.01 & 9.00141 & 9.00002 & 9.00001 & 0.00330 & 0.04116 \\
14 & 0.2 & 0.1 & 0.01 & 9.00143 & 9.00072 & 9.00050 & 0.00339 & 0.06168 \\
15 & 0.2 & 0.05 & 0.01 & 9.00151 & 9.00061 & 9.00042 & 0.00351 & 0.06630 \\
16 & 0.1 & 1 & 0.01 & 9.00500 & 9.00002 & 9.00002 & 0.00126 & 0.03852 \\
17 & 0.1 & 0.1 & 0.01 & 9.00501 & 9.00197 & 9.00114 & 0.00129 & 0.06456 \\
18 & 0.1 & 0.05 & 0.01 & 9.00518 & 9.00334 & 9.00176 & 0.00135 & 0.06948 \\
19 & 0.05 & 1 & 0.01 & 9.01157 & 9.00003 & 9.00002 & 0.00045 & 0.03654 \\
20 & 0.05 & 0.1 & 0.01 & 9.01157 & 9.00281 & 9.00148 & 0.00045 & 0.06468 \\
21 & 0.05 & 0.05 & 0.01 & 9.01169 & 9.00664 & 9.00294 & 0.00045 & 0.06900 \\
22 & 0.2 & 0.05 & 0.03 & 9.00156 & 9.00113 & 9.00072 & 0.00327 & 0.06630 \\
23 & 0.2 & 0.05 & 0.05 & 9.00168 & 9.0012 & 9.00077 & 0.00282 & 0.06630 \\
24 & 0.2 & 0.05 & 0.07 & 9.00192 & 9.00134 & 9.00082 & 0.00216 & 0.06630 \\
25 & 0.2 & 0.05 & 0.09 & 9.00242 & 9.00159 & 9.00091 & 0.00153 & 0.06588 \\
26 & 0.2 & 0.05 & 0.11 & 9.00321 & 9.00202 & 9.00106 & 0.00111 & 0.06558
\end{tabular}

толщины сенсора, но увеличивается почти на порядок и достигает значения 41.37 мкВ при уменьшении толщины поглотителя до 0.05 мкм. Скорость счета не сильно зависит от толщины поглотителя, увеличивается с увеличением толщины сенсора и достигает максимального значения 30.47 ТГц при $Z_{2}=0.05$ и $Z_{3}=1$ мкм. При этих толщинах поглотителя и сенсора $V_{m}=40.9$ мкВ. Такую конструкцию чувствительного элемента можно считать оптимальной для регистрации фотонов с энергией 0.8 эВ.

\section{b. Поглощение фотонов с энергией 0.95 эВ}

В табл. 3 представлены характеристики ТОД при поглощении фотонов с энергией 0.95 эВ в чувствительном элементе $\mathrm{SiO}_{2} / \mathrm{W} / \mathrm{FeSb}_{2} / \mathrm{W} / \mathrm{Al}_{2} \mathrm{O}_{3}$ с толщиной поглотителя $0.2,0.1,0.05$ мкм и толщиной сенсора 1 , 0.1 и 0.05 мкм. По сравнению с предыдущей таблицей в данной таблице добавлен столбец глубины термализации фотона в поглотителе $(h)$, так как в некоторых расчетах этот параметр варьируется. Параметры $T_{b 2 m}$ и $T_{b 4 m}$, во всех расчетах имеют значения много ниже уровня фона и поэтому не представлены в таблице. Данный факт, как и в рассмотренных выше расчетах для фотонов с энергией 0.8 эВ, позволяет сделать вывод, что сигнал детектора будет зависеть только от температуры на границе поглотитель-сенсор.

При термализации фотона с большей энергией в поглотителе чувствительного элемента ТОД выделяется большее количество тепла, что должно приводить к достижению более высоких значений параметров $T_{a 0 m}$, $T_{b 0 m}$ и $T_{c 0 m}$. Также должны достигаться более высокие значения параметра $t_{b}$, так как требуется больше времени для рассасывания выделенного тепла и спада $T_{a} 0$ до фонового значения. И все это видно при сравнении данных табл. 3 и 2.

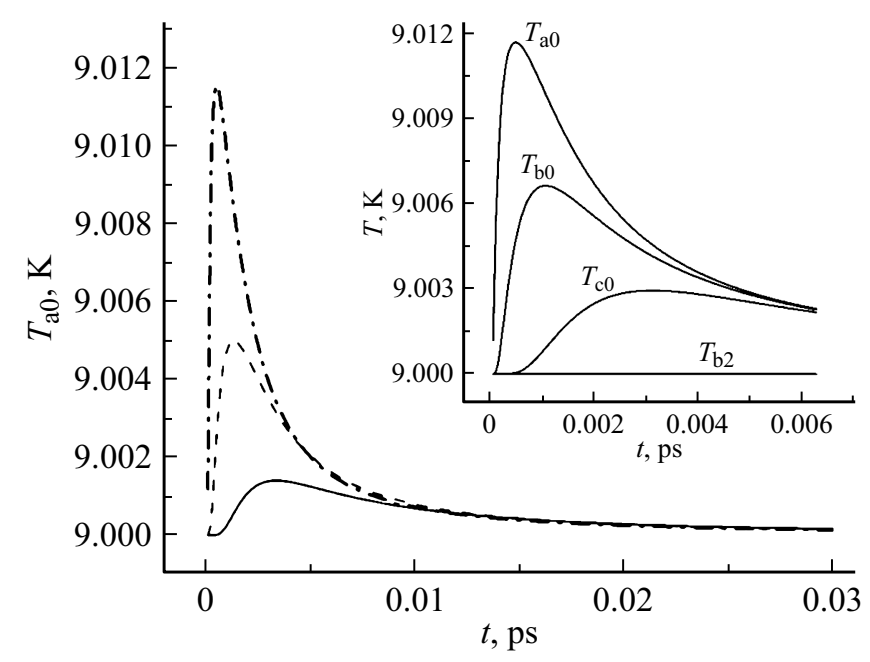

Рис. 3. Зависимость $T_{a 0}(t)$ по расчетам чувствительных элементов с $Z_{2}=1$ мкм и $Z_{3}$, равной 0.2 мкм (сплошная линия), 0.1 мкм (пунктир) и 0.05 мкм (штрихпунктир) после поглощения фотона с энергией 0.95 эВ. На вставке временна́я зависимость параметров $T_{a 0}, T_{b 0}, T_{b 2}$ и $T_{c 0}$ по данным расчета 21.

Сравнение характеристик ТОД трех групп расчетов с номерами $13-15,16-18$ и $19-21$ для толщин $Z_{2}$ соответственно $0.2,0.1$ и 0.05 мкм показывает, что с уменьшением толщины $Z_{3}$ от 1 до 0.05 мкм несколько увеличиваются все полученные в результате расчетов параметры. Параметры, характеризующие максимальную температуру на границах слоев чувствительного элемента, увеличиваются в значительно большей степени с уменьшением толщины $Z_{2}$. Временна́я зависимость температуры в различных областях чувствительного элемента ТОД при поглощении фотонов с энергией 0.95 эВ приведена на рис. 3. На рисунке показаны графики временно́й зависимости температуры $T_{a 0}$ расчетов 13, 16 и 19 для трех различных значений толщины $Z_{2}$ 


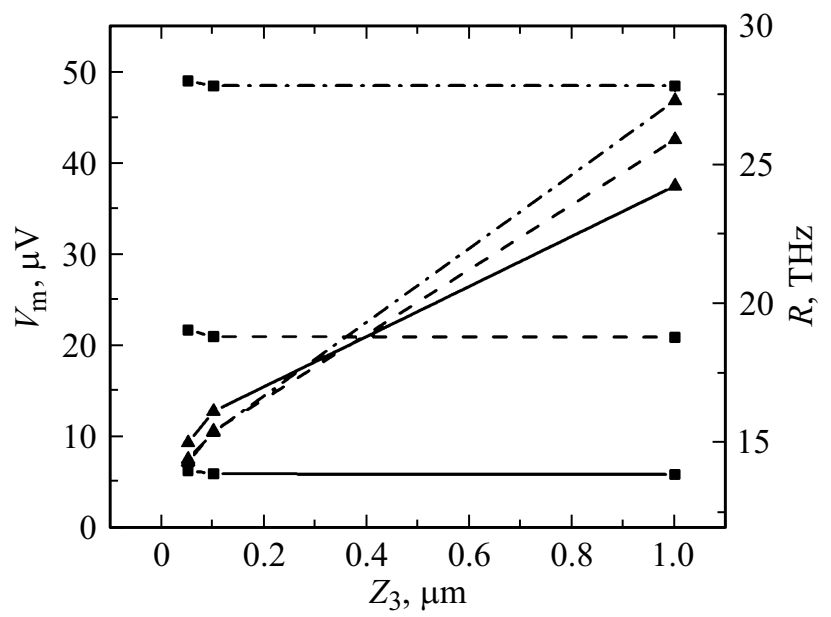

Рис. 4. Зависимость $V_{m}$ (квадраты) и $R$ (треугольники) от толщины $Z_{3}$ для значений $Z_{2}$, равных 0.2 мкм (сплошная линия), 0.1 мкм (пунктирная линия) и 0.05 мкм (штрихпунктирная линия).

при одинаковой толщине $Z_{3}$. Можно видеть, что с уменьшением $Z_{2}$ параметр $T_{a 0 m}$ увеличивается, а $t_{m}-$ уменьшается. Амплитуда сигнала тем выше, чем меньше толщина $Z_{2}$ на всем протяжении процесса вплоть до спада температуры $T_{a 0}$ до уровня фона.

На вставке рис. 3 представлены полученные в результате расчета 21 временнб́е зависимости температуры в различных областях чувствительного элемента ТОД. На границе $B$ достигаются довольно высокие значения $T_{b 0}$, однако температура $T_{b 2}$ остается значительно ниже уровня фона.

Данные расчетов 13-21 позволяют определить значения параметров $V_{m}$ и $R$ после поглощения фотона с энергией 0.95 эВ в чувствительном элементе ТОД различной геометрии (рис. 4). Сравнение графиков на рис. 4 и 2 показывает, что в случае поглощения фотонов с энергией 0.95 эВ достигаются более высокие значения максимального сигнала и более низкая скорость счета по сравнению с поглощением 0.8 эВ фотонов в чувствительном элементе с одинаковой геометрией.

\section{с. Термализация фотонов с энергией 0.95 эВ на различной глубине}

В расчетах табл. 3 с номерами 15, 22-26 приведены данные термализации фотонов с энергией 0.95 эВ на глубине $0.01-0.11$ мкм в вольфрамовом поглотителе толщиной 0.2 мкм $\left(Z_{3}=0.05\right.$ мкм). Из данных таблицы видно, что чем ближе зона термализации фотона к сенсору, тем выше температуры $T_{a 0 m}, T_{b 0 m}$ и $T_{c 0 m}$, тем меньше параметр $t_{m}$, а параметр $t_{b}$ изменяется незначительно. Данные результаты не удивляют, так как чем ближе к сенсору термализуется фотон, тем больше тепла доходит до границ $A, B$ и $C$, тем выше параметры $T_{a 0 m}, T_{b 0 m}$ и $T_{c 0 m}$, а параметр $t_{m}$ меньше, так как выделенному фотоном теплу надо пройти в поглотителе меньшее расстояние, чтобы достичь границы $A$. Параметр $t_{b}$ не претерпевает больших изменений, так как обусловливается в первую очередь геометрией всего чувствительного элемента.

Рассчитанные по данным этих расчетов значения параметров $V_{m}$ и $R$ представлены на рис. 5. В области значений глубины термализации от 0.01 до 0.05 мкм, где вероятность поглощения фотона с энергией 0.95 эВ составляет 99.8\%, параметр $R$ не меняется, а изменение $V_{m}$ составляет всего $10 \%$. Это меньше, чем разница параметров $V_{m}$ расчетов $4(0.8 \mathrm{\jmath})$ и $15(0.95 \mathrm{\jmath B})$ для чувствительного элемента одинаковой геометрии.

Важными характеристиками однофотонных детекторов являются время ожидания сигнала $\left(t_{d}\right)$ и его разброс (временно́й джиттер), который определяется шириной на полувысоте функции распределения $t_{d}$. Оценим временной джиттер ТОД, используя вероятность поглоще-

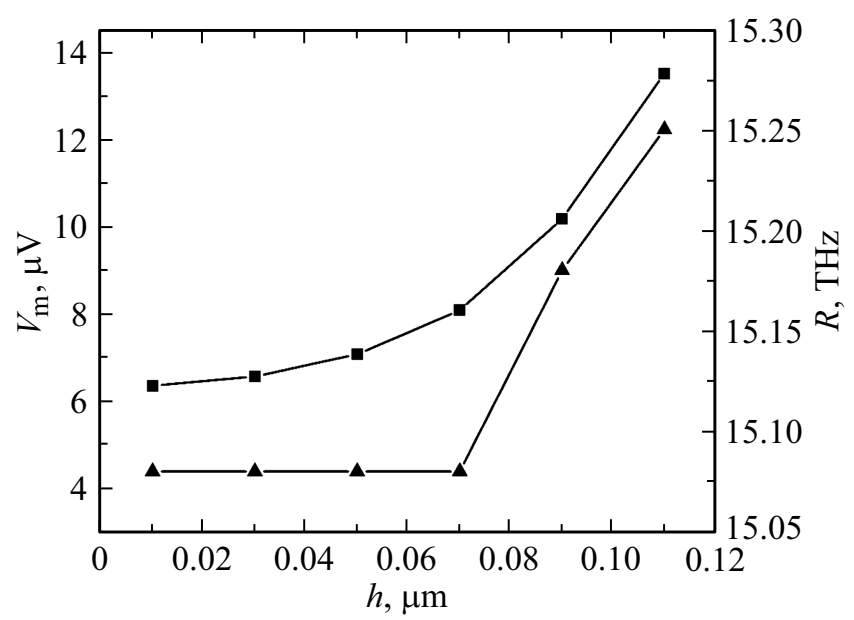

Рис. 5. Зависимость параметров $V_{m}$ (квадраты) и $R$ (треугольники) от глубины зоны термолизации.

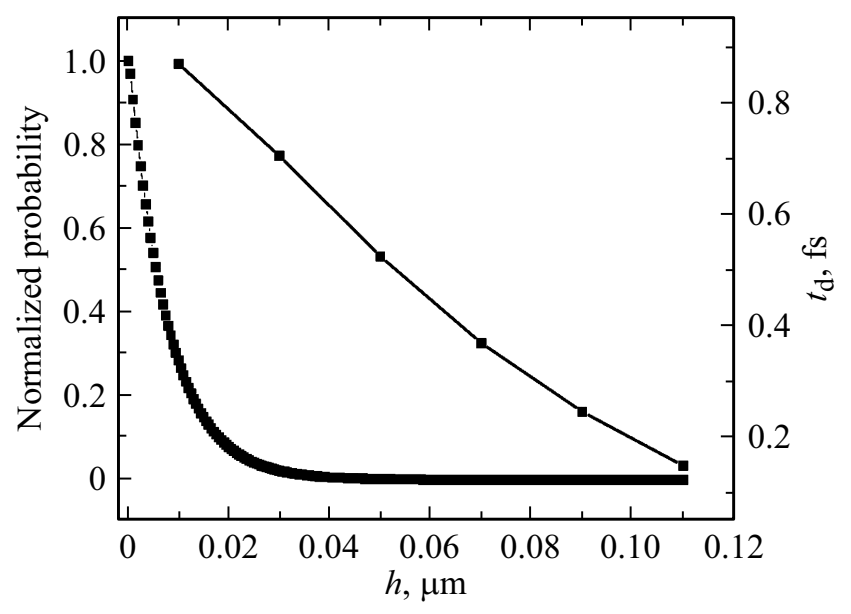

Рис. 6. Нормированная вероятность поглощения фотонов с энергией 0.95 э на определенной глубине в вольфраме и время ожидания сигнала. 
ния фотонов с энергией 0.95 эВ в вольфраме. Время ожидания сигнала определялось как время достижения параметром $T_{a 0}$ значения, в 2 раза превышающего уровень фона. Время ожидания сигнала и нормированная вероятность поглощения фотонов с энергией 0.95 эВ на глубине $h$ в вольфраме приведены на рис. 6. Ширина на полувысоте зависимости вероятности поглощения фотонов от глубины $h$ составляет 0.0055 мкм. Как видно из рис. 6, зависимость времени задержки сигнала от $h$ практически линейна. Из наклона этой зависимости нетрудно рассчитать, что при разнице глубины термализации фотона 0.0055 мкм временна́я задержка будет отличаться на $4.6 \cdot 10^{-2}$ фс. Это и есть временной джиттер нашего детектора для регистрации фотонов с энергией 0.95 эВ, обусловленный различной вероятностью поглощения фотона на разной глубине поглотителя.

\section{4. Заключение}

Результаты компьютерного моделирования процессов распространения тепла в четырехслойном чувствительном элементе ТОД с полупроводниковым $\mathrm{FeSb}_{2}$ сенсором после поглощения фотонов с энергией 0.8 и 0.95 эВ показали, что достигаются высокие характеристики.

Из данных табл. 2 и 3 следует, что чувствительный элемент с поглотителем толщиной 0.05 мкм (расчеты 9-12, 19-21) обеспечивает сигнал в 97 и более раз выше уровня фона. Это обеспечивает 100\% эффективность регистрации поглощенного фотона. Согласно расчетам, эффективность поглощения 0.8 и 0.95 эВ фотонов в вольфраме толщиной 0.05 мкм составляет 99.7 и $99.8 \%$. Антиотражающий слой $\mathrm{SiO}_{2}$ в рассматриваемой области энергий фотонов обеспечивает эффективность оптической связи $\sim 99 \%$. Соответственно, системная эффективность детектирования ТОД, которая является произведением этих трех составляющих, также будет близка к $100 \%$.

Использование $\mathrm{FeSb}_{2}$ в качестве материала сенсора позволяет получать сигнал на уровне десятков микровольт, который для фотонов с энергией 0.8 эВ в 40 раз превышает сигнал четырехслойного чувствительного элемента с $\mathrm{CeB}_{6}$ сенсором [19]. Такой сигнал в эксперименте можно регистрировать без применения сложных систем усиления, которые в свою очередь могут увеличивать уровень шумов. Одновременно достигаются высокие временны́е параметры: задержка сигнала меньше фемтосекунды, временной джиттер $4.6 \cdot 10^{-2}$ фс и терагерцовая скорость счета. Детекторы с такими характеристиками востребованы в различных областях науки и могут найти широкое применение в технологиях будущего.

\section{Благодарности}

Автор выражает благодарность А.С. Кузаняну и В.Р. Никогосяну за помощь в работе и полезные обсуждения.

\section{Финансирование работы}

Исследование выполнено при финансовой поддержке Государственного комитета по науке МОН РА в рамках научного проекта № 18T-2F134.

\section{Конфликт интересов}

Автор заявляет об отсутствии конфликта интересов.

\section{Список литературы}

[1] C.J. Chunnilall, I.P. Degiovanni, S. Kuck, I. Muller, A.G. Sinclair. Opt. Eng., 53 (8), 081910 (2014).

[2] M.D. Eisaman, J. Fan, A. Migdall, S.V. Polyakov. Rev. Sci. Instrum., 82, 071101 (2011).

[3] R.H. Hadfield. Nature Photonics, 3, 696 (2009).

[4] E. Martinenghi, L. Di Sieno, D. Contini, M. Sanzaro, A. Pifferi, A. Dalla Mora. Rev. Sci. Instrum., 87, 073101 (2016).

[5] B. Korzh, T. Lunghi, K. Kuzmenko, G. Boso, H. Zbinden. J. Mod. Opt., 62 (14), 1151 (2015).

[6] K.D. Irvin. Scientific American, 295, 86 (2006).

[7] G.N. Gol'tsman, K. Smirnov, P. Kouminov, B. Voronov, N. Kaurova, V. Drakinsky, J. Zhang, A. Verevkin, R. Sobolewski. IEEE Trans. Appl. Supercond., 13 (2), 192 (2003).

[8] H. Zhang, L. Xiao, B. Luo, J. Guo, L. Zhang, J. Xie. J. Phys. D, 53, 013001 (2020).

[9] D. Van Vechten, K. Wood, G. Fritz, J. Horwitz, A. Gyulamiryan, A. Kuzanyan, V. Vartanyan, A. Gulian. Nucl. Instrum. Meth. Phys. Res. A, 444, 42 (2000).

[10] K. Wood, G. Gilbert, A. Gulian, D. van Vechten. US Patent 6710343 (2004).

[11] A. Gulian, K. Wood, D. Van Vechten, G. Fritzdet. J. Mod. Opt., 51 (9-10), 1467 (2004).

[12] А.С. Кузанян, А.А. Кузанян, В.Р. Никогосян, В.Н. Гурин, М.П. Волков. ФТП, 51 (7), 908 (2017).

[13] A.A. Kuzanyan, V.R. Nikoghosyan, A.S. Kuzanyan. J. Cont. Phys., 53 (4), 338 (2018).

[14] A.A. Kuzanyan, V.R. Nikoghosyan, A.S. Kuzanyan. J. Cont. Phys., 53 (1), 73 (2018).

[15] А.С. Кузанян, А.А. Кузанян, В.Н. Гурин, М.П. Волков, В.Р. Никогосян. ФТП, 53 (5), 689 (2019).

[16] A.A. Kuzanyan, V.R. Nikoghosyan, A.S. Kuzanyan. J. Cont. Phys., 52 (3), 249 (2017).

[17] A.S. Kuzanyan. Sensors Transducers, 217 (11), 28 (2017).

[18] A.A. Kuzanyan, A.S. Kuzanyan, V.R. Nikoghosyan. J. Cont. Phys., 54 (2), 175 (2019).

[19] A.A. Kuzanyan, V.R. Nikoghosyan, A.S. Kuzanyan. IEEE Sens. J., 20 (6), 3040 (2020).

[20] A.A. Kuzanyan, V.R. Nikoghosyan, A.S. Kuzanyan. SPIE Proc., 9504 (2015).

[21] G. Carini Jr., G. Carini, D. Cosio, G. D’Angelo, F. Rossi. Philos. Mag., 96, 761 (2016).

[22] https://nanoheat.stanford.edu/sites/default/files/publications/ A33.pdf

[23] M.S. Figueira, J. Silva-Valencia, R. Franco. Eur. Phys. J. B, 85 (203), 1 (2012).

[24] A. Bentien, S. Johnsen, G.K.H. Madsen, B.B. Iversen, F. Steglich. Europhys. Lett., 80 (1), 17008 (2007). 
[25] T.R. Waite, R. S. Craig, W. E. Wallace. Phys. Rev., 104 (5), 1240 (1956).

[26] http://www.efunda.com/materials/elements/TC_Table.cfm?Elment_ID $=$ W

[27] G.T. Furukawar, T.B. Douglasr, R.E. McCoske,jr, D.C. Ginnings. J. Res. Nat. Bur. Stand., 57, 67 (1956).

[28] http://www.phys.ufl.edu/ireu/IREU2013/pdf_reports/Allen_ Scheie_FinalReport.pdf

[29] M. Caloz, M. Perrenoud, C. Autebert, B. Korzh, M. Weiss, C. Schönenberger, R.J. Warburton, H. Zbinden, F. Bussiéres. Appl. Phys. Lett., 112, 061103 (2018).

[30] W.S.M. Werner, K. Glantschnig, C. Ambrosch-Draxl. J. Phys. Chem. Ref. Data, 38 (4), 1013 (2009).

Редактор Г.А. Оганесян

\section{Semiconductor sensor of a thermoelectric single-photon detector for near-infrared radiation registration}

A.A. Kuzanyan

Institute for Physical Research, National Academy of Sciences of Armenia, 0203 Ashtarak, Armenia

Abstract We proposed the design of a four-layer detection pixel of the single-photon thermoelectric detector with semiconductor $\mathrm{FeSb}_{2}$ sensor. The processes of heat propagation in a detection pixel after the absorption of a photon were studied using computer simulation. The calculations were based on the equation of heat propagation from a limited volume using the three-dimensional matrix method for differential equations. The temporal dependences of the detector signal amplitude were calculated for various thicknesses of the detection pixel's layers and the following parameters were determined: signal delay, timing jitter, maximum signal value, time to reach the maximum signal, decay time and count rate. It was proved that a detector with such a detection pixel can provide detection efficiency above $95 \%$ for near-infrared photons. At the same time, the terahertz count rate was achieved. 